\title{
Cerebral Infarct/Intracranial Cerebrovascular Disease
}

When imaging patients for intracranial cerebral vascular disease, the goals are (1) to assess the degree of parenchymal injury and identify intraparenchymal hemorrhage; (2) to determine if there are areas of altered perfusion that may be at risk for future injury; and (3) to assess the intracranial arteries (patency as well as direction of flow). The corresponding sequences are (1) $T_{2}$ fast spin echo (FSE) and fluid attenuated inverson recovery (FLAIR) to detect acute, subacute, and chronic injury, as well as diffusion-weighted imaging to detect hyperacute injury and to determine age of injury, and gradient-echo imaging to detect intracranial hemorrhages; (2) perfusion-weighted imaging; followed by (3) 3-D time-of-flight MR angiography (TOF-MRA) to examine vascular patency, and phase-contrast MRA (PC-MRA) to provide information regarding the direction of flow in major vessels. The Basic Protocol can be used to evaluate stable patients with acute, subacute, or chronic cerebrovascular symptoms. For a more tailored protocol in cases of hyperacute strokes or cerebrovascular symptoms in unstable patients, see the Alternate Protocol.

\section{STANDARD IMAGING FOR CEREBRAL INFARCT/INTRACRANIAL CEREBROVASCULAR DISEASE}

In order to perform the recommended perfusion and diffusion studies, a scanner with echo planar capabilities is required (Table A1.1.1). MR angiography and the standard anatomical MR imaging sequences included in the protocol do not require these faster gradients. The parameters given here are optimized for a 1.5 Tesla (T) General Electric (GE) LX system with version 8.35 software and may need modification for different software versions, field strengths, or manufacturers.

Data processing is required for the following three sequences described in this protocol, (1) 3-D TOF MRA, (2) diffusion-weighted imaging, and (3) perfusion-weighted imaging. For optimal assessment of the volumetric 3-D TOF MRA data, separate volumes of interest (VOI) of the left anterior, right anterior, and posterior circulation should be created. Images from $15^{\circ}$ rotations about the vertical axis should be saved. An additional anterior circulation volume of interest should include both anterior cerebral arteries and the region of the anterior communicating artery for visual inspection. Images from $15^{\circ}$ rotations about the horizontal axis should also be saved. The authors recommend maximum intensity projection (MIP) over volume and surface reconstructions for assessment of vascular patency, as algorithms in many software packages for surface and volume reconstructions may smooth out and therefore obscure regions of arterial narrowing. All of the authors' MRA data is processed on a General Electric Medical Systems (GEMS)

Table A1.1.1 Equipment Requirements for Cerebral Vascular Assessment

\begin{tabular}{ll}
\hline Coil type & Quadrature head coil \\
Gradient coil strength & $25 \mathrm{mT} / \mathrm{m}$ (or whatever the system permits) \\
Slew rate & $\geq 120 \mathrm{~T} / \mathrm{m} / \mathrm{sec}$ \\
Cardiac gating & Not necessary \\
Peripheral gating & Not necessary \\
Respirator & If required by patient \\
Oxygen & If required by patient \\
Motion cushions & Useful \\
Use of contrast agents & Yes \\
\hline
\end{tabular}

Contributed by P. Ellen Grant, Pamela W. Schaefer, and R. Gilberto Gonzalez

Current Protocols in Magnetic Resonance Imaging (2001) A1.1.1-A1.1.13

Copyright $\odot 2001$ by John Wiley \& Sons, Inc.
BASIC PROTOCOL

Intracranial Arterial Disease A1.1.1 
Advantage Windows Workstation version 3.1P using standard GEMS Advantage Windows software or FuncTool version 1.9M.

Diffusion imaging uses a spin echo (SE) echo planar sequence with additional bipolar gradients around the $180^{\circ} \mathrm{RF}$ (radio frequency) pulse to increase the sensitivity to molecular motion. This includes blood flow and water diffusion. Water diffusion is highly anisotropic in the brain due to the highly ordered, tightly packed, and anisotropic alignment of white matter tracts. When the bipolar gradient is oriented parallel to the white matter tracts, water diffuses readily and signal loss occurs. If the gradients are oriented perpendicular to the tracts in a direction of restricted water diffusion, no signal loss occurs. Thus, the calculation of an apparent diffusion coefficient must balance these anisotropic effects by measuring signal changes in $\geq 3$ directions. In fact, a full description would require the measurement of the full diffusion tensor but this is not usually used clinically. The raw data from the diffusion-weighted sequence must be processed to provide both the trace diffusion-weighted image (commonly referred to as the DWI) and the apparent diffusion coefficient (ADC) map. Most diffusion sequences, including the GE diffusion sequence, process the data automatically to provide the DWI and the spin echo (SE) echo-planar (EP) $T_{2}$ sequence but most do not automatically provide the ADC map. The standard GE diffusion sequence can be processed on an Advantage Windows Workstation version 3.1P using FuncTool version 1.9M to obtain ADC maps. With the authors' custom diffusion sequence, both DWI and ADC maps are automatically obtained.

The perfusion of blood through the brain parenchyma can be monitored by tracking the first passage of a rapid, well defined bolus of intravenously administered MR contrast agent through the brain using echo planar imaging. There is a magnetic susceptibility induced signal reduction as the bolus of paramagnetic contrast agent, confined to the vascular compartment, passes through the brain. The time series data from the perfusion sequence must be processed in order to obtain relative cerebral blood volume (rCBV), time to peak (TTP), relative cerebral blood flow (rCBF), and mean transit time (MTT) maps. The most accurate method for obtaining these parameters is to convert plots of signal intensity versus time to plots of $\Delta R_{2}{ }^{*}=\Delta\left(1 / T_{2}{ }^{*}\right)$ versus time. A filter is used to decrease high frequency noise prior to conversion of signal intensity to $R_{2}{ }^{*}$. Next, to determine the bolus dispersion in the smaller arterial feeders and tissue, the bolus dispersion that occurs in the larger feeding artery is removed by deconvolving the tissue curve with the arterial input curve, typically the middle cerebral artery. Although the deconvolution step is not needed for the calculation of rCBV or TTP, the authors typically calculate all parameters after the deconvolution. In order to reduce errors from motion artifact, time points with excessive motion are deleted prior to processing. This perfusion data analysis is custom software. However, perfusion analysis software is also available with FuncTool version $1.9 \mathrm{M}$ that will give similar results.

For perfusion-weighted imaging in adults, the use of a double dose of contrast agent injected by an MR compatible injection pump, such as a MEDRAD injector, at a rate of $5 \mathrm{ml} / \mathrm{sec}$ through an 18-G angiocatheter is recommended. If an injection pump is not available, perfusion sequences can still be performed with a hand injector using a 3-way stopcock to inject a contrast bolus followed rapidly by a saline flush. The contrast agent should be injected $\sim 10 \mathrm{sec}$ after the start of the scan to provide enough images prior to contrast agent arrival to determine the precontrast baseline and to provide enough images after the contrast agent arrival to see the entire first pass of the bolus.

NOTE: Be sure that technologists and nurses have immediate access to any emergency equipment that may be relevant to a given study, or that may be needed for a particular patient, such as crash carts or oxygen. 


\section{Materials}

Normal saline $(0.9 \% \mathrm{NaCl})$, sterile

Gadolinium-based MR contrast agent (e.g., Magnevist, Omniscan, or Prohance)

\section{Set up patient and equipment}

1. Interview (screen) the patient to ensure that he or she has no contraindications such as cardiac pacemakers or other implants containing ferromagnetic materials. Also be sure to find out if the patient has any health conditions that may require the presence of special emergency equipment during the scanning procedure, or necessitate any other precautions. Do not forget to ask if the patient has any drug allergies, and document them.

Generally, standard screening forms are used for all patients scanned in a magnetic resonance system.

The presence of any ferromagnetic metals may be a health hazard to the patient when he or she is inside the magnet, and will also affect the imaging. If in doubt as to the exact composition of the items, it is best to exclude patients with any metal implants; see Shellock (1996) for discussion of what implants may be safely scanned using magnetic resonance.

Patients may be accompanied into the magnet room by a friend or family member, who can sit in the room during the scan and comfort the patient as needed. This companion must be screened as well to ensure the absence of loose metal objects on the body or clothing.

2. If the procedure is a research protocol, have the patient sign any necessary consent form.

3. Have the patient remove all jewelry and change into a gown to eliminate any metal that might be found in clothing.

4. Have the patient wash off any mascara and other makeup to avoid local tissue heating and image artifacts.

5. Inform the patient about what will occur during the procedure, what he or she will experience while in the magnet, and how to behave, including the following:

a. If earphones or headphones are used to protect the ears from the loud sounds produced by the gradients, the patient will be asked to wear these, but will be able to communicate with you at any time during the imaging.

b. The patient will be given a safety squeeze-bulb or similar equipment to request assistance at any time (demonstrate how this works).

c. For good results the patient should not talk, and should avoid or minimize swallowing or other movement, during each scan-i.e., as long as the banging sounds continue. Between scans, talking and swallowing are allowed in most cases, but should be avoided when comparative positional studies are being performed; the patient will be informed when this is the case.

d. Nevertheless, the patient may call out at any time if he or she feels it necessary.

6. If an $18-\mathrm{G}$ i.v. is not already in place, an i.v. with an $18-\mathrm{G}$ angiocatheter should be started.

7. Have the patient mount onto the table. Either before or right after the patient lies down, set up any triggering devices or other monitoring equipment that is to be used.

8. Connect the MR compatible injection pump that is cleared of air and loaded with a double dose of contrast agent and with saline flush, to the patient. Do a small test injection to ensure that the i.v. is working properly so that contrast agent can be injected when the perfusion sequence is performed.

Intracranial Arterial Disease

A1.1.3 
9. Center the patient in a head or neck coil at the region where the key information is desired. Make sure that the head and neck are constrained to prevent motion, especially if high-resolution scans are to be run.

Generally the patient's head is fixed so that the head is horizontal (not tilted) and the neck and head lie along the axis of the patient table; other positions may be appropriate depending on the needs at hand.

Most scanners have a special neck coil for MRA; otherwise, a head coil should be used and the patient placed as far in as possible so that the bifurcation of the common carotid artery into the internal and external carotid arteries can be imaged.

10. If needed, place a pillow or other support under the knees to make the patient more comfortable.

11. Use the centering light focused on the nasion to position the patient and put him or her into the center of the magnet.

Once this step has been performed, so long as the patient does not move on the table, the table itself can be moved and then replaced in the same position as before without jeopardizing the positioning of one scan relative to another.

12. If the patient is unable to hold still, provide an appropriate sedative.

13. Program the $5 \mathrm{ml} / \mathrm{sec}$ injection rate for both contrast agent and saline, contrast agent dose required (double dose by weight), saline flush required (typically $40 \mathrm{ml}$ ), and 10 -sec time delay. Start the saline running to keep the vein open and arm the injection pump. Do not inject the contrast agent until the perfusion sequence.

\section{Sequence 1: Rapid sagittal $T_{1}$ scout}

14. To determine the patient's position, a fast sagittal scout scan is performed using the imaging sequence in Table A1.1.2. (Some medical centers may prefer a rapid three-plane scout instead of this multislice sagittal scout.)

\section{Sequence 2: Transverse diffusion}

15. From the sagittal scout, select the image through the center of the brain to set up the locations for the transverse diffusion sequence. The transverse images should begin at the foramen magnum and end at the top of the brain. Set up imaging parameters as shown in Table A1.1.3.

16. Warn the patient that the sequence results in loud beeping (banging) noises and begin the scan.

\section{Data processing and viewing for sequence 2}

17. Most diffusion sequences will perform a minimum of 4 sequences: three with orthogonal diffusion gradient directions and one with a minimal or no diffusion gradient.

Often, a fifth set of images is also provided, which combines the 3 orthogonal gradient images to produce a set of images whose signal intensity is not affected by the diffusion direction. This is the DWI. In order to obtain apparent diffusion coefficient (ADC) maps, the images may need further processing. On a GE system, the ADC maps can be obtained by processing on the Advantage Window Workstation version 3.1P using FuncTool version $1.9 M$.

Cerebral Infarct/ Intracranial Cerebrovascular Disease A1.1.4

\section{Sequence 3: Transverse $T_{2}{ }^{*}$-weighted gradient echo}

18. To detect hemorrhages, this gradient echo sequence should be performed. From the sagittal scout, select the image through the center of the brain to set up the locations for the transverse $T_{2}{ }^{*}$-weighted gradient echo sequence. The locations should be the 
Table A1.1.2 Rapid Sagittal $T_{1}$ Scout

\begin{tabular}{ll}
\hline Patient position & Supine \\
Scan type & Gradient echo \\
Imaging plane (orientation) & Sagittal \\
Central slice or volume center & Nasion \\
Echo time $\left(T_{\mathrm{E}}\right)$ & Minimum (at least $4.1 \mathrm{msec})$ \\
Receiver bandwidth (RBW) & $15.6 \mathrm{kHz}$ \\
Repeat time $\left(T_{\mathrm{R}}\right)$ & $100 \mathrm{msec}$ \\
Flip angle $(\mathrm{FA})$ & $60^{\circ}$ \\
Fields of view $\left(\mathrm{FOV}_{\mathrm{x}}, \mathrm{FOV} \mathrm{y}\right)$ & $240 \mathrm{~mm}, 240 \mathrm{~mm}$ \\
Resolution $(\Delta x, \Delta y)$ & $0.94 \mathrm{~mm}, 1.25 \mathrm{~mm}$ \\
Number of data points collected $\left(N_{\mathrm{x}}, N_{\mathrm{y}}\right)$ & 256,192 \\
Display matrix $\left(D_{\mathrm{x}}, D_{\mathrm{y}}\right)$ & 256,256 \\
Slice thickness $(\Delta z)$ & $7 \mathrm{~mm}$ \\
Number of slices & 7 \\
Slice gap & $2 \mathrm{~mm}$ \\
Number of excitations $(\mathrm{NEX})$ & 1 \\
Number of acquisitions $\left(N_{\mathrm{acq}}\right)$ & 1 \\
Read direction & Superior-inferior \\
Scan time & $16 \mathrm{sec}$ \\
\hline
\end{tabular}

Table A1.1.3 Transverse Diffusion

\begin{tabular}{ll}
\hline Patient position & Supine \\
Scan type & Single shot SE-EPI, diffusion \\
Imaging plane (orientation) & Transverse \\
Pulse sequence database (PSD) & epi2NV \\
Central slice or volume center & Nasion \\
Echo time $\left(T_{\mathrm{E}}\right)$ & $101 \mathrm{msec}$ \\
Repeat time $\left(T_{\mathrm{R}}\right)$ & $7500 \mathrm{msec}$ \\
Flip angle $(\mathrm{FA})$ & $90^{\circ}$ \\
Fields of view $\left(\mathrm{FOV}_{\mathrm{x}}, \mathrm{FOV}_{\mathrm{y}}\right)$ & $220 \mathrm{~mm}, 220 \mathrm{~mm}$ \\
Resolution $(\Delta x, \Delta y)$ & $1.72 \mathrm{~mm}, 1.72 \mathrm{~mm}$ \\
Number of data points collected $\left(N_{\mathrm{x}}, N_{\mathrm{y}}\right)$ & 128,128 \\
Display matrix $\left(D_{\mathrm{x}}, D_{\mathrm{y}}\right)$ & 128,128 \\
Slice thickness $(\Delta z)$ & $5 \mathrm{~mm}$ \\
Number of slices & 24 \\
Slice gap & $1 \mathrm{~mm}$ \\
Number of excitations $(\mathrm{NEX})$ & 3 \\
Number of acquisitions $\left(N_{\mathrm{acq}}\right)$ & 1 \\
Read direction & Right-left \\
Saturation pulses & Fat saturation $($ automatic with EPI) \\
Control variables $(\mathrm{CV})$ & Ramp sampling $=1$, burst \\
& sampling =0 \\
$b$-value & $1000 \mathrm{sec} / \mathrm{mm}^{2}$ \\
Scan time & $2 \mathrm{~min}, 38 \mathrm{sec}$ \\
\hline
\end{tabular}


Table A1.1.4 Transverse $T_{2}$-Weighted Gradient Echo

\begin{tabular}{ll}
\hline Patient position & Supine \\
Scan type & 2-D gradient echo \\
Imaging plane (orientation) & Transverse \\
Variable bandwidth & Yes \\
Central slice or volume center & Nasion \\
Echo time $\left(T_{\mathrm{E}}\right)$ & $25 \mathrm{msec}$ \\
Receiver bandwidth $(\mathrm{RBW})$ & $15.63 \mathrm{kHz}$ \\
Repeat time $\left(T_{\mathrm{R}}\right)$ & $750 \mathrm{msec}$ \\
Flip angle $(\mathrm{FA})$ & $20^{\circ}$ \\
Fields of view $(\mathrm{FOV}, \mathrm{FOV}$ & $\mathrm{y})$ \\
Resolution $(\Delta x, \Delta y)$ & $240 \mathrm{~mm}, 240 \mathrm{~mm}$ \\
Number of data points collected $\left(N_{\mathrm{x}}, N_{\mathrm{y}}\right)$ & $0.94 \mathrm{~mm}, 1.25 \mathrm{~mm}$ \\
Display matrix $\left(D_{\mathrm{x}}, D_{\mathrm{y}}\right)$ & 256,192 \\
Slice thickness $(\Delta z)$ & 256,256 \\
Number of slices & $5 \mathrm{~mm}$ \\
Slice gap & 23 \\
Number of excitations $(\mathrm{NEX})$ & $1 \mathrm{~mm}$ \\
Number of acquisitions $\left(N_{\mathrm{acq}}\right)$ & 2 \\
Read direction & 1 \\
Flow compensation & Anterior-posterior \\
Scan time & Yes \\
\hline
\end{tabular}

Table A1.1.5 Transverse $T_{2}$-Weighted FSE

Patient position

Scan type

Imaging plane (orientation)

Central slice or volume center

Variable bandwidth

Pulse sequence database (PSD)

Echo time $\left(T_{\mathrm{E}}\right)$

Receiver bandwidth (RBW)

Echo train length (ETL)

Repeat time $\left(T_{\mathrm{R}}\right)$

Flip angle (FA)

Fields of view $\left(\mathrm{FOV}_{\mathrm{x}}, \mathrm{FOV}_{\mathrm{y}}\right)$

Resolution $(\Delta x, \Delta y)$

Number of data points collected $\left(N_{\mathrm{x}}, N_{\mathrm{y}}\right)$

Display matrix $\left(D_{\mathrm{x}}, D_{\mathrm{y}}\right)$

Slice thickness $(\Delta z)$

Number of slices

Slice gap

Number of excitations (NEX)

Number of acquisitions $\left(N_{\text {acq }}\right)$

Read direction

Flow compensation

Extended dynamic range (EDR)

Scan time
Supine

Fast spin echo

Transverse

Nasion

Yes

FSE-XL

$102 \mathrm{msec}$

$11.36 \mathrm{kHz}$

12

$6000 \mathrm{msec}$

$90^{\circ}$

$240 \mathrm{~mm}, 240 \mathrm{~mm}$

$0.94 \mathrm{~mm}, 1.25 \mathrm{~mm}$

256, 192

256, 256

$5 \mathrm{~mm}$

28

$1 \mathrm{~mm}$

2

1

Anterior-posterior

Yes

Yes

$2 \mathrm{~min}, 48 \mathrm{sec}$

\section{Cerebral Infarct/ \\ Intracranial Cerebrovascular Disease}

A1.1.6 
Table A1.1.6 Transverse FLAIR

\begin{tabular}{ll}
\hline Patient position & Supine \\
Scan type & Inversion recovery fast spin echo \\
Imaging plane (orientation) & Transverse \\
Variable bandwidth & Yes \\
Central slice or volume center & Nasion \\
Echo time $\left(T_{\mathrm{E}}\right)$ & $140 \mathrm{msec}$ \\
Receiver bandwidth (RBW) & $20.83 \mathrm{kHz}$ \\
Echo train length $(\mathrm{ETL})$ & 14 \\
Repeat time $\left(T_{\mathrm{R}}\right)$ & $10,000 \mathrm{msec}$ \\
Inversion time $\left(T_{\mathrm{I}}\right)$ & $2200 \mathrm{msec}$ \\
Flip angle $(\mathrm{FA})$ & $180^{\circ}$ \\
Fields of view $(\mathrm{FOV}, \mathrm{x}, \mathrm{FOV}$ & $\mathrm{y})$ \\
Resolution $(\Delta x, \Delta y)$ & $240 \mathrm{~mm}, 240 \mathrm{~mm}$ \\
Number of data points collected $\left(N_{\mathrm{x}}, N_{\mathrm{y}}\right)$ & $0.94 \mathrm{~mm}, 1.25 \mathrm{~mm}$ \\
Display matrix $\left(D_{\mathrm{x}}, D_{\mathrm{y}}\right)$ & 256,192 \\
Slice thickness $(\Delta z)$ & 256,256 \\
Number of slices & $5 \mathrm{~mm}$ \\
Slice gap & 14 \\
Number of excitations $(\mathrm{NEX})$ & $1 \mathrm{~mm}$ \\
Number of acquisitions $\left(N_{\mathrm{acq}}\right)$ & 1 \\
Read direction & 2 \\
Scan time & Anterior-posterior \\
\hline
\end{tabular}

same as those chosen for the transverse diffusion sequence. Set up the imaging parameters as shown in Table A1.1.4.

19. Warn the patient that the sequence is starting and begin the scan.

\section{Sequence 4: Transverse $T_{2}$-weighted FSE}

20. From the sagittal scout, select the image through the center of the brain to set up the locations for the transverse $T_{2}$-weighted FSE sequence. The locations should be the same as those chosen for the transverse diffusion sequence. Set up the imaging parameters as shown in Table A1.1.5.

21. Warn the patient that the sequence is starting and begin the scan.

\section{Sequence 5: Transverse FLAIR}

22. From the sagittal scout, select the image through the center of the brain to set up the locations for the transverse FLAIR sequence. The locations should be the same as those chosen for the transverse diffusion sequence. Set up the imaging parameters as shown in Table A1.1.6.

23. Warn the patient that the sequence is starting and begin the scan.

\section{Sequence 6: Transverse 3-D TOF MRA}

24. From the sagittal scout, select the image through the center of the brain to set up the locations for the transverse 3-D TOF MRA sequence. Set up the imaging parameters as shown in Table A1.1.7. Two slabs are posted on the midline sagittal image, centered at the top of the sella turcica.

25. Warn the patient that the sequence is starting and begin the scan.

Intracranial Arterial Disease

A1.1.7 
Table A1.1.7 Transverse 3-D TOF MRA

\begin{tabular}{ll}
\hline Patient position & Supine \\
Scan type & 3-D short- $T_{\mathrm{R}}$ gradient echo \\
Imaging plane (orientation) & Transverse \\
Variable bandwidth & Yes \\
Central slice or volume center & Nasion \\
Echo time $\left(T_{\mathrm{E}}\right)$ & $3.2 \mathrm{msec}$ \\
Receiver bandwidth $(\mathrm{RBW})$ & $15.6 \mathrm{kHz}$ \\
Repeat time $\left(T_{\mathrm{R}}\right)$ & $33 \mathrm{msec}$ \\
Flip angle $(\mathrm{FA})$ & $20^{\circ}$ \\
Fields of view $\left(\mathrm{FOV}, \mathrm{x}, \mathrm{FOV}_{\mathrm{y}}\right)$ & $160 \mathrm{~mm}, 160 \mathrm{~mm}$ \\
Resolution $(\Delta x, \Delta y)$ & $0.63 \mathrm{~mm}, 0.84 \mathrm{~mm}$ \\
Number of data points collected $\left(N_{\mathrm{x}}, N_{\mathrm{y}}\right)$ & 256,192 \\
Display matrix $\left(D_{\mathrm{x}}, D_{\mathrm{y}}\right)$ & 256,256 \\
Slice thickness $(\Delta z)$ & $1.4 \mathrm{~mm}$ \\
Number of slices per slab & 32 \\
Number of slabs & 2 \\
Slab overlap & 4 slices \\
Slice gap & $0 \mathrm{~mm}$ \\
Number of excitations $(\mathrm{NEX})$ & 1 \\
Number of acquisitions $\left(N_{\mathrm{acq}}\right)$ & 1 \\
Read direction & Anterior-posterior \\
Flow compensation & Yes \\
ZIP 512 & Yes \\
ZIP 2 & Yes \\
Extended dynamic range $(\mathrm{EDR})$ & Yes \\
Saturation pulses & Superior \\
Magnetization transfer & Yes \\
Vascular options & Reprojections $=19^{\circ}$ \\
& Collapse $=$ on \\
Scan time & Ramp pulse $=$ inferior to superior \\
\hline & $6 \mathrm{~min}, 32 \mathrm{sec}$ \\
\hline &
\end{tabular}

Cerebral Infarct/ Intracrania Cerebrovascular Disease

A1.1.8
Data processing and viewing for sequence 6

26. Once the transverse partitions from the 3-D TOF MRA are obtained, volumes of interest (VOI) that select out the posterior circulation, the right anterior circulation, and the left anterior circulation should be selected, and the maximum intensity projections (MIPs) of these 3 VOIs rotated through $360^{\circ}$ about the vertical, saving images at $15^{\circ}$ intervals. A fourth VOI that selects out both anterior circulations, should be rotated through $360^{\circ}$ about the horizontal, saving images at $15^{\circ}$ intervals.

\section{Sequence 7: Transverse 2-D phase contrast}

27. From the sagittal scout, select the image through the center of the brain to set up the locations for the transverse 2-D PC MRA sequence. Set up the imaging parameters as shown in Table A1.1.8. Three slices are posted on the midline sagittal image, centered at the top of the sella turcica.

28. Warn the patient that the sequence is starting and begin the scan. 


\begin{tabular}{ll}
\hline Patient position & Supine \\
Scan type & $\begin{array}{l}\text { 2-D Vascular phase contrast } \\
\text { gradient echo }\end{array}$ \\
Imaging plane (orientation) & Transverse \\
Central slice or volume center & Nasion \\
Echo time $\left(T_{\mathrm{E}}\right)$ & $6.7 \mathrm{msec}$ \\
Repeat time $\left(T_{\mathrm{R}}\right)$ & $33 \mathrm{msec}$ \\
Flip angle $(\mathrm{FA})$ & $20^{\circ}$ \\
Fields of view $(\mathrm{FOV}, \mathrm{x}, \mathrm{FOV} \mathrm{y})$ & $220 \mathrm{~mm}, 165 \mathrm{~mm}$ \\
Resolution $(\Delta x, \Delta y)$ & $0.86 \mathrm{~mm}, 1.03 \mathrm{~mm}$ \\
Number of data points collected $\left(N_{\mathrm{x}}, N_{\mathrm{y}}\right)$ & 256,160 \\
Display matrix $\left(D_{\mathrm{x}}, D_{\mathrm{y}}\right)$ & 256,256 \\
Slice thickness $(\Delta z)$ & $15 \mathrm{~mm}$ \\
Number of slices & 3 \\
Slice gap & 0 mm \\
Number of excitations $(\mathrm{NEX})$ & 4 \\
Number of acquisitions $\left(N_{\mathrm{acq}}\right)$ & 3 \\
Read direction & Anterior-posterior \\
Flow compensation & Yes \\
Slice series & Sequential \\
Vascular options & Flow reconstruction type $=$ phase \\
& difference \\
& Velocity encoding $=80 \mathrm{~cm} / \mathrm{sec}$ \\
Scan time & Acquired flow directions $=$ all \\
\hline & Collapse $=$ on \\
& Flow analysis $=$ off \\
& Additional flow images $=\mathrm{R} / \mathrm{L}$, \\
& A/P, S/I, MAG \\
& 2 min, 6 sec \\
\hline
\end{tabular}

\section{Sequence 8: Transverse perfusion}

29. Using the transverse FSE $T_{2}$-weighted images, find the slice location where the middle cerebral arteries are best seen. Using the imaging parameters as shown in Table A1.1.9, set up the transverse images starting at the same location that the MCA's (middle cerebral artery) were identified on the FSE $T_{2}$-weighted images. Typically, only 10 or 11 slices can be obtained in the given $T_{\mathrm{R}}$ interval. The slice thickness and gap can be modified so that the perfusion study covers any diffusion abnormality.

30. Check that the injection pump is ready to inject the contrast agent and that the injection of the contrast agent is set to start $10 \mathrm{sec}$ after the scan begins.

31. Warn the patient that the sequence is starting and that part of the way through the scan the i.v. injection will occur. Warn the patient that a cool sensation may be felt during the injection. Begin the scan.

Two doses of contrast agent $(0.2 \mathrm{mmol} / \mathrm{kg})$ are given here.

\section{Process data and view for sequence 8}

32. Process the perfusion images.

The perfusion images can be processed on an Advantage Windows Workstation version $3.1 P$ using FuncTool version 1.9M to give maps of relative cerebral blood volume $(r C B V)$, relative cerebral blood flow $(r C B F)$, and mean transit time (MTT).

Intracranial Arterial Disease

A1.1.9 


\begin{tabular}{ll}
\hline Patient position & Supine \\
Scan type & Single shot SE-EPI \\
Imaging plane (orientation) & Transverse \\
Variable bandwidth & Yes \\
Pulse sequence database (PSD) & epi2perf \\
Central slice or volume center & Nasion \\
Echo time $\left(T_{\mathrm{E}}\right)$ & $65 \mathrm{msec}$ \\
Repeat time $\left(T_{\mathrm{R}}\right)$ & $1520 \mathrm{msec}$ \\
Flip angle $(\mathrm{FA})$ & $90^{\circ}$ \\
Fields of view $(\mathrm{FOV}, \mathrm{FOV}$ & $\mathrm{F})$ \\
Resolution $(\Delta x, \Delta y)$ & $220 \mathrm{~mm}, 220 \mathrm{~mm}$ \\
Number of data points collected $\left(N_{\mathrm{x}}, N_{\mathrm{y}}\right)$ & $1.72 \mathrm{~mm}, 1.72 \mathrm{~mm}$ \\
Display matrix $\left(D_{\mathrm{x}}, D_{\mathrm{y}}\right)$ & 128,128 \\
Slice thickness $(\Delta z)$ & 128,128 \\
Number of slices & 5 or $6 \mathrm{~mm}$ \\
Slice gap & 11 \\
Number of excitations $(\mathrm{NEX})$ & $1 \mathrm{~mm}$ \\
Number of acquisitions $\left(N_{\mathrm{acq}}\right)$ & 1 \\
Read direction & 1 \\
Slice location & Left-right \\
Multi-phase & Inferior slice to include MCA \\
Slice series & 46 phases per location, minimum \\
Control variables $(\mathrm{CV})$ & delay between acquisitions \\
Scan time & Interleaved \\
\hline
\end{tabular}

\section{RAPID IMAGING FOR ACUTE CEREBRAL INFARCT}

When an acute arterial stroke is suspected, MR imaging with diffusion and perfusion imaging provides information that is necessary for rapid, optimal patient treatment. If the patient is unstable, if the patient is an intra-arterial thrombolysis candidate, or if the patient must be squeezed into an already over-booked MR schedule, imaging is kept to a minimum. The choice of $T_{2}$-weighted sequence for sequence 3 depends on user preference. Arguments for $T_{2}$-weighted FSE sequence include shorter scan time with good spatial resolution, for FLAIR, increased sensitivity to cortical and periventricular hyperintensity, and for gradient echo, increased sensitivity to blood products.

\section{Set up patient and equipment}

1. Use the same equipment and perform the same equipment and patient set up as in Basic Protocol, steps 1 to 13.

\section{Sequence 9: Rapid sagittal scout}

2. Run rapid sagittal scout (see Basic Protocol, sequence 1, step 14).

\section{Sequence 10: Transverse diffusion}

3. Run transverse diffusion sequence (see Basic Protocol, sequence 2, steps 15 to 17).

\section{Cerebral Infarct/ \\ Intracranial Cerebrovascular Disease}


Sequence 11: Transverse $T_{2}$-weighted FSE

4. Run transverse $T_{2}$-weighted FSE sequence (see Basic Protocol, sequence 4, steps 20 and 21).

\section{Sequence 12: Transverse perfusion}

5. Run transverse perfusion sequence (see Basic Protocol, sequence 8, steps 29 and 32).

\section{Sequence 13: 2-D phase contrast MRA}

6. Run transverse 2-D phase contrast MRA sequence (see Basic Protocol, sequence 7, steps 27 and 28).

\section{COMMENTARY}

\section{Background Information}

Strokes are the third leading cause of death and the major cause of adult long-term disability. The concept of "Brain Attack" has emphasized the importance of rapid treatment in stroke management. To enable rapid treatment, rapid detection of an acute stroke is essential. With the high sensitivity of diffusion-weighted imaging to acute stroke (Gonzalez et al., 1999), it has become the mainstay of stroke evaluation. In animal models, diffusion-weighted imaging can detect acute ischemic injury as early as 10 min after arterial occlusion (Kucharczyk et al., 1991). In the authors' clinical experience, diffusion-weighted imaging can detect hyperacute infarcts in humans as early as 30 min after a witnessed event, well before the standard $T_{2}$-weighted or FLAIR imaging becomes abnormal. In the subacute stages, when there is mixture of chronic and recent events, diffusionweighted imaging is helpful in determining which of many bright areas on $T_{2}$-weighted images is the more recent lesion. The DWI, when combined with the ADC map, can also help determine the age of the lesion (Warach et al., 1995; Burdette et al., 1998; 1999).

Perfusion-weighted imaging provides complementary information about blood volume in and blood flow through the tissue. This information may be helpful in assessing the risk for infarct growth and the degree of hemodynamic compromise (Sorensen et al., 1996; Rordorf et al., 1998; Sorensen et al., 1999). In most clinical settings where imaging is performed at least a few hours after the event, the area of decreased rCBV best predicts the final infarct size (Sorensen et al., 1999). When the areas of decreased relative cerebral blood flow and elevated mean transit time are significantly larger than the diffusion abnormality, the ipsilateral ICA (internal carotid artery) or MCA is typically occluded. When such areas of "mismatch" between relative blood flow and diffusion exist, there is an increased risk that the infarct will increase in size, although the mechanism of this interval growth is not yet clear.

The 3-D TOF MRA is useful for determining the patency of major vessels and the degree of intracranial atherosclerotic disease. The 2-D PC MRA gives a lower resolution evaluation of vessel patency but is significantly faster and therefore more appropriate when time is an issue. It is adequate to detect vessel cut-off. The 2-D PC MRA also provides information regarding the direction of flow in major vessels that can help determine routes of collateral flow in cases with vascular occlusion.

The standard anatomical MR sequences, namely the FLAIR and $T_{2}$-weighted FSE sequences, allow us to assess the condition of the brain parenchyma and determine if there is evidence of old ischemic events. The $T_{2}{ }^{*}$ weighted gradient echo sequence is probably as accurate as computed tomography (CT) at determining if there is evidence of blood products in the setting of acute stroke (Perl et al., 1999).

\section{Critical Parameters and Troubleshooting}

For diffusion-weighted imaging, the " $b$ value" is a critical parameter in determining ADC values for different tissues, particularly when the $b$-value is between 1000 and 2000 $\mathrm{sec} / \mathrm{mm}^{2}$ (Le Bihan et al., 1991; Mulkern et al., 1999). The human studies on stroke imaging in the literature have all been done with $b$-values close to $1000 \mathrm{sec} / \mathrm{mm}^{2}$ and the protocol described here uses a $b$-value of $1000 \mathrm{sec} / \mathrm{mm}^{2}$. At this $b$-value, there is a good signal to noise ratio (SNR) and the ADC values of gray and white matter are essentially equal. With increasing $b$-values, the SNR drops and ADC values of gray and white matter start to diverge. Many systems now have the capability of doing diffusion-weighted imaging at $b$-values $>1000$
Intracranial Arterial Disease A1.1.11 
$\mathrm{sec} / \mathrm{mm}^{2}$ but extreme caution must be used when interpreting these results. The $T_{\mathrm{E}}$ is also an important parameter in diffusion-weighted imaging. To optimize SNR, the shortest possible $T_{\mathrm{E}}$ is used. $T_{\mathrm{R}}$ has no effect on image contrast. With increasing or decreasing $T_{\mathrm{R}}$, the number of slices obtained increases or decreases.

For perfusion-weighted imaging, a spin echo echo-planar sequence or a gradient echo echo-planar sequence can be used. In this protocol, a spin echo echo-planar sequence is chosen because it has a greater contribution from small vessels (Boxerman et al., 1995). The trade-off is a decreased change in signal with the bolus of contrast compared to the gradient echo sequence. In order to improve the signal change, a double dose of contrast agent is used for the spin echo sequence that results in a signal drop of at least $10 \%$. In perfusion weighted imaging, increasing $T_{\mathrm{R}}$ increases the number of slices available but at the cost of increasing the temporal spacing between points on the signal intensity versus time curve. Short spacing in time enables better characterization of the bolus. The $T_{\mathrm{R}}$ chosen in this sequence is what the authors determined to be the best compromise between these two factors. The $T_{\mathrm{E}}$ is also an important parameter to optimize. Varying the $T_{\mathrm{E}}$ not only alters the SNR but also changes the contribution of different vessel sizes on relative blood volume and relative blood flow calculations (Boxerman et al., 1995). The authors have chosen a $T_{\mathrm{E}}$ of $65 \mathrm{msec}$ to best optimize SNR and the contribution of small diameter vessels.

Important basic parameters in MR angiography include $T_{\mathrm{R}}$, flip angle, and $T_{\mathrm{E}}$. The $T_{\mathrm{R}}$ is chosen to be long enough to avoid saturation of flowing blood but short enough to improve contrast by saturation of background tissue. $T_{\mathrm{E}}$ is typically minimized to minimize flow related dephasing. The flip angle must be optimized to be short enough to avoid saturation of small vessels but long enough to give sufficient suppression of background tissue. Most vendors have ramped flip angles where the flip angle is gradually increased in the direction of the flowing blood to minimize saturation. Interpolation, performed by zero filling the acquisition matrix (number of data points collected) in $k$-space, is helpful in reducing partial volume averaging effects. Typically, the authors interpolate in both the slice select and the phase encoding directions. In cases with high-grade stenosis, turbulence may result in an over estimation of the degree of vascular narrowing. Decreasing the $T_{\mathrm{R}}$ and flip angle can minimize these effects at the cost of decreased background suppression. Other alternatives include contrast agent administration or black blood techniques (see Table A7.4.10).

\section{Anticipated Results}

Diffusion-weighted imaging should allow detection of ischemic necrosis as early as 30 min and at least as long as 10 days after the ischemic event. Perfusion-weighted imaging provides complementary information on the relative blood volume and blood flow that helps determine areas of hemodynamic compromise. The MRA allows assessment of the degree of underlying cerebral vascular disease and can detect areas of vascular occlusion.

\section{Time Considerations}

The full assessment of patients at risk for an ischemic event often includes neck MR angiography as well as the Basic Protocol described above. This results in a study of at least $45 \mathrm{~min}$ in length. When time is crucial for optimal patient treatment, as in candidates for intra-arterial thrombolysis, the Alternate Protocol is used to minimize imaging time yet provides the critical information required for decisions on urgent patient management.

\section{Literature Cited}

Boxerman, J.L., Hamberg, L.M., Rosen, B.R., and Weisskoff, R.M. 1995. MR contrast due to intravascular magnetic susceptibility perturbations. Magn. Reson. Med. 34:555-566.

Burdette, J.H., Ricci, P.E., Petitti, N., and Elster, A.D. 1998. Cerebral infarction: Time course of signal intensity changes on diffusion-weighted MR images. Am. J. Roentgenol. 171:791-795.

Burdette, J.H., Elster, A.D., and Ricci, P.E. 1999. Acute cerebral infarction: Quantification of spindensity and $\mathrm{T}_{2}$ shine-through phenomena on diffusion-weighted MR images. Radiology 212:333-339.

Gonzalez, R.G., Schaefer, P.W., Buonanno, F.S., Schwamm, L.H., Budzik, R.F., Rordorf, G., Wang, B., Sorensen, A.G., and Koroshetz, W.J. 1999. Diffusion-weighted MR imaging: Diagnostic accuracy in patients imaged within 6 hours of stroke symptom onset. Radiology 210:155162.

Kucharczyk, J., Mintorovitch, J., Asgari, H.S., and Moseley, M. 1991. Diffusion/perfusion MR imaging of acute cerebral ischemia. Magn. Reson. Med. 19:311-315.

Le Bihan, D., Moonen, C.T., van Zijl, P.C., Pekar, J., and DesPres, D. 1991. Measuring random microscopic motion of water in tissues with MR imaging: A cat brain study. J. Comput. Assisted Tomogr. 15:19-25. 
Mulkern, R.V., Gudbjartsson, H., Westin CF, Zengingonul HP, Gartner W, Guttmann CR, Robertson RL, Kyriakos W, Schwartz R, Holtzman D, Jolesz FA, Maier SE. 1999. Multicomponent apparent diffusion coefficients in human brain. N.M.R. Biomed. 12:51-62.

Perl, J., 2nd, Tkach, J.A., Porras-Jimenez, M., Lieber, M., Obuchowski, N., Ross, J.S., Ding, X.P., Ruggieri, P.M., Shearer, D.M., Khajavi, K., and Masaryk, T.J. 1999. Hemorrhage detected using MR imaging in the setting of acute stroke: An in vivo model. Am. J. Neuroradiol. 20:1863-1870.

Rordorf, G., Koroshetz, W.J., Copen, W.A., Cramer, S.C., Schaefer, P.W., Budzik, R.F. Jr., Schwamm, L.H., Buonanno, F., Sorensen, A.G., and Gonzalez, G. 1998. Regional ischemia and ischemic injury in patients with acute middle cerebral artery stroke as defined by early diffusionweighted and perfusion-weighted MRI. Stroke 29:939-943.

Shellock, F.G. 1996. Pocket Guide to MR Procedures and Metallic Objects. Lippincott-Raven, Philadelphia.

Sorensen, A.G., Buonanno, F.S., Gonzalez, R.G., Schwamm, L.H., Lev, M.H., Huang-Hellinger, F.R., Reese, T.G., Weisskoff, R.M., Davis, T.L.,
Suwanwela, N., Can, U., Moreira, J.A., Copen, W.A., Look, R.B., Finklestein, S.P., Rosen, B.R., and Koroshetz, W.J. 1996. Hyperacute stroke: Evaluation with combined multisection diffusion-weighted and hemodynamically weighted echo-planar MR imaging. Radiology 199:391401.

Sorensen, A.G., Copen, W.A., Ostergaard, L., Buonanno, F.S., Gonzalez, R.G., Rordorf, G., Rosen, B.R., Schwamm, L.H., Weisskoff, R.M., and Koroshetz, W.J. 1999. Hyperacute stroke: Simultaneous measurement of relative cerebral blood volume, relative cerebral blood flow, and mean tissue transit time. Radiology 210:519527.

Warach, S., Gaa, J., Siewert, B., Wielopolski, P., and Edelman, R.R. 1995. Acute human stroke studied by whole brain echo planar diffusionweighted magnetic resonance imaging. Ann. Neurol. 37:231-241.

Contributed by P. Ellen Grant, Pamela W. Schaefer, and R. Gilberto Gonzalez Massachusetts General Hospital Boston, Massachusetts
Intracranial Arterial Disease 